\title{
One-step nucleic acid amplification testing in medullary thyroid cancer lymph nodes: a case series
}

\author{
Krzysztof Kaczka ${ }^{1}$, Wojciech Fendler ${ }^{2}$, Maciej Borowiec ${ }^{2}$, Wojciech Młynarski², \\ Katarzyna Paduszynska ${ }^{1}$, Anna Grzegory ${ }^{1}$, Lech Pomorski ${ }^{1}$
}

\author{
${ }^{1}$ Department of General and Oncological Surgery, University Hospital and Education \\ Centre of Medical University of Lodz, Lodz, Poland \\ ${ }^{2}$ Department of Pediatrics, Oncology, Hematology and Diabetology, Medical University \\ of Lodz, Lodz, Poland
}

Submitted: 10 April 2013

Accepted: 2 July 2013

Arch Med Sci 2015; 11, 1: 137-141

DOI: $10.5114 /$ aoms.2015.49206

Copyright $\odot 2015$ Termedia \& Banach

\section{Abstract}

Introduction: Locoregional relapse in medullary thyroid cancer (MTC) may be caused by nodal micrometastases. Medullary thyroid cancer lymph nodes have not yet been evaluated by one-step nucleic acid amplification (OSNA). Therefore, the aim of this study was to detect MTC cells by OSNA in cervical lymph nodes and compare the obtained outcomes with conventional histopathology.

Material and methods: Twenty-one randomized, unenlarged lymph nodes from 5 patients with MTC were examined by histopathology and OSNA. Lymph nodes were divided into four representative blocks by a sterile, single use, special cutting device in the same way as in the clinical protocol study performed by Tsujimoto et al. Two blocks were used for histopathology and immunohistochemistry, 2 for OSNA.

Results: Positive results of histopathology and OSNA were revealed in $4 \mathrm{pa}$ tients. The outcomes of OSNA and histopathology were corresponding in 3 patients. Positive histopathology results of 2 lymph nodes from 2 patients were confirmed by OSNA. In 1 patient there were only negative results of both examinations. One-step nucleic acid amplification failed to detect metastasis in 1 lymph node in 2 patients although it did not change the TNM status in these patients. There were no false positive results in the OSNA test.

Conclusions: One-step nucleic acid amplification may be an alternative method to histopathology in detecting nodal involvement in MTC. Further studies should evaluate the sensitivity and specificity of OSNA and the impact on staging in MTC.

Key words: medullary thyroid cancer, lymph nodes, histopathology, onestep nucleic acid amplification.

\section{Introduction}

Medullary thyroid carcinoma (MTC) is a neuroendocrine tumor originating from $C$ cells of the thyroid. Medullary thyroid carcinoma is a rather rare carcinoma. It accounts for $5 \%$ to $10 \%$ of thyroid carcinoma and for $0.2 \%$ to $0.4 \%$ of all goiter patients [1]. Medullary thyroid carcinoma may be either sporadic (about 75\%) or hereditary (about 25\%), associated with genetic syndromes - multiple endocrine neoplasia type 2A (MEN2A) and $2 B$ as well as familial medullary thyroid carcinoma $[1,2]$. Lymph node

\author{
Corresponding author: \\ Krzysztof Kaczka \\ Department of General \\ and Oncological Surgery \\ University Hospital \\ and Education Centre \\ Medical University of Lodz \\ 251 Pomorska St \\ 92-231 Lodz, Poland \\ Phone: +48 503068786 \\ E-mail: krzysztofkaczka@ \\ poczta.fm
}


metastases are common in MTC $[1,3,4]$. In sporadic $\mathrm{MTC}$, they are observed in the central compartment in $50 \%$ of patients, in the ipsilateral compartment in $57 \%$ and in the contralateral in $28 \%$. In hereditary MTC, lymph node metastases are identified in the central compartment in $45 \%$ of patients, in the ipsilateral compartment in $36 \%$, and in the contralateral compartment in $19 \%$ [3]. Lymph node metastases in MTC significantly worsen the prognosis [3]. Surgery is the only curative treatment modality for MTC. There are many national and institutional guidelines that standardize MTC treatment and optimize outcomes [5]. Survival time in MTC is relatively long, but locoregional relapse is frequent [6-8]. This might be explained by micrometastases in cervical lymph nodes that are not found by conventional histology.

In the treatment of MTC, thyroidectomy with central lymph node dissection is almost universally accepted, but the extent of lateral lymphadenectomy is controversial $[1,9]$. Some propose performing modified radical neck dissection initially even in the absence of any suspicious lymph nodes [3]. Such an aggressive approach can cause more complications and lengthen the time of operation, but it eliminates the disease completely and results in better prognosis. Therefore, it is important to identify patients with a poor prognosis in order to undertake a more aggressive approach, and perform more careful follow-up to provide early surgery of the relapse. There is a need to look for methods other than histopathology of lymph node metastases detection [10, 11]. Nowadays, molecular techniques are developing rapidly. Real time-polymerase chain reaction (RT-PCR) and one-step nucleic acid amplification (OSNA) are the most common. Medullary thyroid carcinoma is an epithelial cell derived cancer, so like other such cancers it can be examined by OSNA. According to the best of our knowledge, MTC lymph nodes have not yet been evaluated by OSNA. We suppose it will be useful to check the feasibility of OSNA tests in medullary thyroid cancer. Perhaps, OSNA will detect micrometastases in lymph nodes, which are not found in conventional histopathology. They could be responsible for the presence of residual disease and elevated calcitonin levels found postoperatively.

Therefore, the aim of this study was to detect MTC cells by OSNA in cervical lymph nodes and compare the obtained outcomes with conventional histopathology.

\section{Material and methods}

The study was done in agreement with the guidelines of the Ethical Commission of the Medical University of Lodz and in compliance with the Declaration of Helsinki.

\section{Characteristics of patients}

Twenty-one randomized, unenlarged cNO lymph nodes from 5 patients (4 men, 1 woman, mean age 42.8) with MTC were examined by histopathology and OSNA. The diameter of lymph nodes ranged from $8 \mathrm{~mm}$ to $11 \mathrm{~mm}$. One patient (BR) underwent total thyroidectomy with appropriate lymphadenectomy (Table I). The other 4 patients underwent lymphadenectomy because they were reoperated on due to nodal recurrence. Nodal recurrence in 4 patients appeared from 8 to 22 months after the primary operation. These patients have not been treated by chemotherapy and radiotherapy.

The type of operation was in accordance with the guidelines of the Polish Society of Surgeons and the Polish Society of Oncological Surgery [12]. Performing the OSNA test did not change the surgical procedure.

\section{Lymph node samples division}

The lymph nodes for the study were prepared according to the protocol proposed by Tsujimoto et al. [13]. All lymph node were separated from fat tissue before division. Each of the 21 randomized lymph nodes were divided into representative parts and were assessed using histopathology with immunohistochemistry and OSNA. First, they were divided into four blocks by a sterile, single use, special cutting device in the same way as in the clinical protocol study performed by Tsujimoto et al. In our procedure blocks a and c were used for OSNA. Blocks $b$ and $d$ were used for histopathology with immunohistochemistry.

\section{Histopathological}

and immunohistochemical procedure

Blocks $b$ and $d$ were fixed in formalin, embedded in paraffin and sectioned for further studies. The sections were stained with hematoxylin and eosin and observed under a light microscope. Immunohistochemical staining for calcitonin was performed on formalin-fixed, paraffin-embedded sections with anticalcitonin antibodies (Dako, Germany). Presence of calcitonin was visualized with EnVision System (Dako, Germany).

All specimens were examined by 2 pathologists. One-step nucleic acid amplification results were unknown to the pathologists performing histopathology.

\section{One-step nucleic acid amplification procedure}

The OSNA test for blocks a and c of each lymph node consisted of homogenization of tissue in an mRNA-stabilizing solution and subsequent amplification. It was done automatically by reverse tran- 
Table I. Results of histopathology and OSNA. (0) - negative histopathology, (1) - positive histopathology

\begin{tabular}{|c|c|c|c|c|}
\hline Sample & Patient & Histopathology & OSNA & CK19 mRNA copy number/ $\mu \mathrm{l}$ \\
\hline 1 & MA & 1 & 1 & $1.3 \times 10^{3}$ \\
\hline 2 & & 0 & 0 & $<2.5 \times 10^{2}$ \\
\hline 3 & & 1 & 1 & $1.8 \times 10^{3}$ \\
\hline 4 & KJ & 0 & 0 & $<2.5 \times 10^{2}$ \\
\hline 5 & & 0 & 0 & $<2.5 \times 10^{2}$ \\
\hline 6 & & 0 & 0 & $<2.5 \times 10^{2}$ \\
\hline 7 & $\mathrm{BR}$ & 1 & 0 & $<2.5 \times 10^{2}$ \\
\hline 8 & & 1 & 1 & $3.6 \times 10^{3}$ \\
\hline 9 & & 1 & 2 & $2.3 \times 10^{4}$ \\
\hline 10 & & 0 & 0 & $<2.5 \times 10^{2}$ \\
\hline 11 & & 0 & 0 & $<2.5 \times 10^{2}$ \\
\hline 12 & PP & 1 & 1 & $2.8 \times 10^{2}$ \\
\hline 13 & & 0 & 0 & $<2.5 \times 10^{2}$ \\
\hline 14 & & 1 & 0 & $<2.5 \times 10^{2}$ \\
\hline 15 & & 0 & 0 & $<2.5 \times 10^{2}$ \\
\hline 16 & & 1 & 1 & $1.4 \times 10^{3}$ \\
\hline 17 & $C M$ & 1 & 1 & $4.1 \times 10^{2}$ \\
\hline 18 & & 0 & 0 & $<2.5 \times 10^{2}$ \\
\hline 19 & & 1 & 1 & $6.8 \times 10^{3}$ \\
\hline 20 & & 1 & 1 & $3.8 \times 10^{3}$ \\
\hline 21 & & 0 & 0 & $<2.5 \times 10^{2}$ \\
\hline
\end{tabular}

scription loop-mediated isothermal amplification (RT-LAMP) of CK19 mRNA in the RD-100i detection engine (Sysmex) without prior mRNA isolation and purification. RD-100i includes a ready-to-use reagent kit (Lynoamp, Sysmex) consisting of the enzyme, primers, nucleotides, buffer necessary for CK19 mRNA amplification and components for assay validation (calibrators, positive and negative controls). The technique uses six primers, which increase the specificity and speed of the reaction. The expression level of CK19 mRNA is detected by real-time monitoring of turbidity changes caused by an increase in the magnesium pyrophosphate concentration, a byproduct of the amplification reaction. Outcomes are automatically characterized by the CK19 mRNA copy number/ $\mu$ l of the original tissue homogenate in accordance with cut-off levels defined by Tsujimoto et al. - nonmetastasis (0) as $<2.5 \times 10^{2}$ copies/ $\mu$ l, micrometastasis (1) as 2.5 $\times 10^{2}$ copies/ $\mu$ to $5 \times 10^{3}$ copies/ $\mu$ l, macrometastasis $(2)>5 \times 10^{3}$ copies $/ \mu$ l [13].

\section{Results}

Positive results of histopathology and OSNA were revealed in 4 patients (Table I). All positive histopathological results were confirmed by immunohistochemistry.

The outcomes of OSNA and histopathology were corresponding in 3 patients. Positive results of histopathology of 2 lymph nodes from 2 patients were confirmed by OSNA. In 1 patient there were only negative outcomes of both examinations. One-step nucleic acid amplification failed to detect metastasis in 1 lymph node in 2 patients (Table I), although it did not change the TNM status in these patients.

There were no false positive results in the OSNA test.

\section{Discussion}

Locoregional relapse occurs in MTC, even when histopathology does not find metastases at the time of the first operation. Probably, this is caused by micrometastases in cervical lymph nodes, which are not detected by the standard histopathologic examination. Serious histopathology is much more sensitive, but it is time- and work-consuming, because it requires the evaluation of many slides, making it impossible to establish it as a routine procedure in many hospital centers. Therefore, mo- 
lecular diagnosis of nodal involvement has been attempted for medullary thyroid cancer. It can be used to make a diagnosis before the operation [14] or as an examination of excised lymph nodes [15]. Weber et al. obtained positive results of CK10 RT-PCR in $19 \%$ of lymph nodes, in which no neoplastic cells were identified by conventional histopathology [15]. These studies suggest that RT-PCR can be a useful tool for lymph node evaluation. It is a pity that the RT-PCR technique requires time to give results, therefore making it inapplicable for intraoperative use. Nowadays, OSNA is a rapidly developing molecular method that can be used as an intraoperative molecular examination. It amplifies cytokeratin 19 mRNA - a typical marker of solid tumors. The material which is examined by this procedure is derived from lymph nodes. At the beginning, the lysate is homogenized in a special buffer, then it is briefly centrifuged. A product, prepared in this way, is subjected to a loop-mediated isothermal amplification with use of reverse-transcription loop-mediated isothermal amplification - RT-LAMP $[13,16]$. Neither purifying mRNA nor changing temperatures during amplification are needed, as it takes place in PCR. It enables one to shorten the time of this process using a constant temperature of $65^{\circ} \mathrm{C}$. The OSNA procedure is automated and can be performed even in an operating theatre. It can differentiate contamination of a few benign epithelial cells using a verified cutoff value.

So far, OSNA has been used most commonly in breast cancer $[17,18]$. However, over time, its utility has been checked in other cancers: colorectal cancer $[19,20]$, gastric cancer [21], head and neck cancers [22, 23], and lung cancer [24]. According to the best of our knowledge, no such study has been performed in MTC. We suppose that it will be useful to introduce this technique in MTC. Perhaps, like other molecular examinations, it will allow one to extract a subgroup of patients upstaged by OSNA: histopathologically negative but molecular biology positive lymph nodes - pNO(mol+) $[25,26]$. Additionally, the sentinel lymph node biopsy is becoming more and more common [27-29]. If it becomes a standard procedure, OSNA rather than RT-PCR could become an alternative to frozen section examination - "classical intraoperative examination". In our preliminary study, 21 randomized, unenlarged lymph nodes from 5 MTC patients were evaluated by OSNA. The results of OSNA and histopathology were exactly the same in 3 of them. One-step nucleic acid amplification failed to detect metastasis in 1 lymph node in 2 patients. This did not change the TNM status in these patients because both examinations showed the same results (positive and negative) in the rest of the lymph nodes. Maybe this was caused by a sampling error - alternate parts of the lymph nodes, which may or may not have con- tained neoplastic cells, were taken for the pathology and the OSNA test. This kind of mistake is impossible to avoid during the feasibility study, because we are obliged to perform both examinations from the same lymph nodes to compare them to each other.

One-step nucleic acid amplification from the tumor of this patient revealed CK19 expression. It is a pity that the whole blocks used for OSNA were homogenized, which made it impossible to perform other tests, especially RT-PCR, on them.

We did not obtain false positive results for OSNA in the whole group of lymph nodes. The application of six primers for CK19 in OSNA allows one to eliminate illegitimate amplification. This phenomenon is the cause of false positive results obtained by RT-PCR. This was described by Bojunga et al. and Aust et al. in thyroid cancer [30, 31]. To reduce the extent of lymphadenectomy we need a molecular examination with the smallest possible number of false positive results. From this point of view, OSNA has a significant advantage over RT-PCR.

Our study has some limitations. Medullary thyroid carcinoma is a rather rare disease, so the number of included subjects is not high. Additionally, the parts of the lymph nodes used for OSNA were homogenized and not used for pathology. Therefore, the results of a study comparing OSNA and histopathology using different pieces of the lymph nodes must include some cases producing discrepant outcomes caused by uneven localization of neoplastic cells. The OSNA method is well studied in breast cancer, and only the patients with this cancer have the whole lymph nodes examined by OSNA without histopathology [32, 33]. One-step nucleic acid amplification uses CK19 as a marker. Maybe other markers, especially calcitonin, will be more suitable for MTC. We think it would be interesting to design a study comparing OSNA with different markers. It is a pity it is possible to perform OSNA only with CK19 as a marker.

In conclusion, we suppose that OSNA might be an alternative method to histopathology in detecting nodal involvement in medullary thyroid cancer. It takes 30-40 min to perform, so it can replace frozen section examinations. It is necessary to perform further studies. These studies should evaluate the sensitivity and specificity of OSNA and the impact on the staging in MTC.

\section{Acknowledgments}

The study received financial support from the Ministry of Science and Higher Education in Poland - grant number N N403 220537.

Wojciech Fendler received financial support from the project "Polish Registry for Pediatric and Adolescent Diabetes - nationwide genetic screening for monogenic diabetes" financed by the Innovative Economy Operational Program. 


\section{Conflict of interest}

The authors declare no conflict of interest.

\section{References}

1. Noullet S, Trésallet C, Godiris-Petit G, et al. Surgical management of sporadic medullary thyroid cancer. J Visc Surg 2011; 148: e244-9.

2. Dralle H, Machens A, Lorenz K. Hereditary thyroid cancer. Chirurg 2008; 79: 1017-28.

3. Scollo C, Baudin E, Travagli JP, et al. Rationale for central and bilateral lymph node dissection in sporadic and hereditary medullary thyroid cancer. J Clin Endocrinol Metab 2003; 88: 2070-5.

4. Kebebew E, Ituarte PH, Siperstein AE, et al. Medullary thyroid carcinoma: clinical characteristics, treatment, prognostic factors, and a comparison of staging systems. Cancer 2000; 88: 1139-48.

5. Panigrahi B, Roman SA, Sosa JA. Medullary thyroid cancer: are practice patterns in the United States discordant from American Thyroid Association guidelines? Ann Surg Oncol 2010; 17: 1490-8.

6. Gülben K, Berberoğlu U, Boyabatli M. Prognostic factors for sporadic medullary thyroid carcinoma. World I Surg 2006; 30: 84-90.

7. Clark, JR, Fridman TR, Odell MJ, et al. Prognostic variables and calcitonin in medullary thyroid cancer. Laryngoscope 2005; 115: 1445-50.

8. Traugott A, Moley JF. Medullary thyroid cancer: medical management and follow-up. Curr Treat Options Oncol 2005; 6: 339-46.

9. Vassiliou I, Tympa A, Arkadopoulos N, et al. Total thyroidectomy as the single surgical option for benign and malignant thyroid disease: a surgical challenge. Arch Med Sci 2013; 21: 74-8.

10. Kupisz K, Stepulak A, Zdunek M, et al. Preliminary results of prognostic significance of proliferating cell nuclear antigen expression in advanced primary larynx carcinomas and lymph nodemetastases. Arch Med Sci 2010; 6: 65-70.

11. Xue N, Huang P, Aronow WS et al. Predicting lymph node status in patients with early gastric carcinoma using double contrast-enhanced ultrasonography. Arch Med Sci 2011; 7: 457-64.

12. Jarząb B, Sporny S, Lange D, et al. Diagnosis and treatment of thyroid cancer - Polish guidelines. Endokrynol Pol 2010; 61: 518-68.

13. Tsujimoto M, Nakabayashi K, Yoshidome K, et al. Onestep nucleic acid amplification for intraoperative detection of lymph node metastasis in breast cancer patients. Clin Cancer Res 2007; 13: 4807-16.

14. Bugalho MJ, Mendonça E, Sobrinho LG. Medullary thyroid carcinoma: an accurate pre-operative diagnosis by reverse transcription-PCR. Eur J Endocrinol 2000; 143: 335-8.

15. Weber T, Amann K, Weckauf H, et al. Detection of disseminated medullary thyroid carcinoma cells in cervical lymph nodes by cytokeratin 20 reverse transcription-polymerase chain reaction. World J Surg 2002; 26: 148-52.

16. Notomi T, Okayama H, Masubuchi H, et al. Loop-mediated isothermal amplification of DNA. Nucleic Acids Res 2000; 28: E63.

17. Tamaki Y, Sato N, Homma K, et al. Routine clinical use of the one-step nucleic acid amplification assay for detection of sentinel lymph node metastases in breast cancer patients: results of a multicenter study in Japan. Cancer 2012; 118: 3477-83.
18. Guillén-Paredes MP, Carrasco-González L, Cháves-Benito $A$, et al. One-step nucleic acid amplification (OSNA) assay for sentinel lymph node metastases as an alternative to conventional postoperative histology in breast cancer: a cost-benefit analysis. Cir Esp 2011; 89: 456-62.

19. Croner RS, Schellerer V, Demund H, et al. One step nucleic acid amplification (OSNA) - a new method for lymph node staging in colorectal carcinomas. J Transl Med 2010; 8: 83.

20. Yamamoto H, Sekimoto M, Oya M, et al. OSNA-based novel molecular testing for lymph node metastases in colorectal cancer patients: results from a multicenter clinical performance study in Japan. Ann Surg Oncol 2012; 18: 1891-8.

21. Yaguchi $Y$, Sugasawa $H$, Tsujimoto $H$, et al. One-step nucleic acid amplification (OSNA) for the application of sentinel node concept in gastric cancer. Ann Surg Oncol 2011; 18: 2289-96.

22. Goda H, Nakashiro K, Oka R, et al. One-step nucleic acid amplification for detecting lymph node metastasis of head and neck squamous cell carcinoma. Oral Oncol 2012; 48: 958-63.

23. Matsuzuka T, Takahashi K, Kawakita D, et al. Intraoperative molecular assessment for lymph node metastasis in head and neck squamous cell carcinoma using onestep nucleic acid amplification (OSNA) assay. Ann Surg Oncol 2012; 19: 3865-70.

24. Inoue M, Hiyama K, Nakabayashi K, et al. An accurate and rapid detection of lymph node metastasis in nonsmall cell lung cancer patients based on one-step nucleic acid amplification assay. Lung Cancer 2012; 78: 212-8.

25. Liefers GJ, Cleton-Jansen AM, van de Velde CJ, et al. Micrometastases and survival in stage II colorectal cancer. N Engl J Med 1998; 339: 223-8.

26. Noura S, Yamamoto H, Ohnishi T, et al. Comparative detection of lymph node micrometastases of stage II colorectal cancer by reverse transcriptase polymerase chain reaction and immunohistochemistry. J Clin Oncol 2002; 20: 4232-41.

27. Kaczka K, Celnik A, Luks B, et al. Sentinel lymph node biopsy techniques in thyroid pathologies - a meta-analysis. Endokrynol Pol 2012; 63: 222-31.

28. Roh JL, Koch WM. Role of sentinel lymph node biopsy in thyroid cancer. Expert Rev Anticancer Ther 2010; 10: 1429-37.

29. Balasubramanian SP, Harrison BJ. Systematic review and meta-analysis of sentinel node biopsy in thyroid cancer. Br J Surg 2011; 98: 334-44.

30. Bojunga J, Röddiger S, Stanisch M, et al. Molecular detection of thyroglobulin mRNA transcripts in peripheral blood of patients with thyroid disease by RT-PCR. Br J Cancer 2000; 82: 1650-5.

31. Aust G, Crisp M, Bösenberg E, et al. Transcription of thyroid autoantigens in non-expressing tissues. Exp Clin Endocrinol Diabetes 1998; 106: 319-23.

32. Ohi Y, Umekita Y, Sagara Y, et al. Whole sentinel lymph node analysis by a molecular assay predicts axillary node status in breast cancer. Br J Cancer 2012; 107: 1239-43.

33. Castellano I, Macrì L, Deambrogio C, et al. Reliability of whole sentinel lymph node analysis by one-step nucleic acid amplification for intraoperative diagnosis of breast cancer metastases. Ann Surg 2012; 255: 334-42. 